\title{
Dual-Process Theory as a Theory of the Classification of Information Processing Acts
}

\author{
- Vitaliy Nadurak -
}

\begin{abstract}
The article proposes a consideration of the dual-process theory of higher cognition as a theory of the classification of acts of information processing. One of the reasons why the dual-process approach has been criticized is the fact that the information processing process can sometimes have characteristics that undermine a clear-cut attribution to one of the two traditionally defined opposite types. To avoid this criticism, it is proposed that the object of classification should not be the processes of information processing, but separate acts of combining two units of information. Unlike a process, a particular act of information processing at a particular moment in time cannot simultaneously have opposite characteristics, nor can it simultaneously have and not have some characteristic. In order to show the qualitative difference between various information processing acts as falling individually into either Type 1 or Type 2 processing, it is proposed to classify them by a feature that is present in one type and absent in another. It is suggested to take conscious control as such a feature. As a result, in the information processing acts corresponding to Type 2 category, units of information are combined in a consciously controlled way, whereas in the acts to be considered as Type 1, those units either already are combined or combine autonomously due to the existence of indirect associative connections.
\end{abstract} Keywords: dual-process theory, information processing, reasoning, intuition, classification.

Published online: 23 December 2021

\section{Introduction}

As Evans and Stanovich noted: "The distinction between two kinds of thinking, one fast and intuitive, the other slow and deliberative, is both ancient in origin and widespread in philosophical and psychological writing." ${ }^{1}$ In recent decades, a large number of studies have appeared that support the idea of two qualitatively distinct kinds of thinking (information processing) and seek to explain them. These studies are often referred to as

\footnotetext{
Vitaliy Nadurak

Department of Philosophy

Sociology and Religious Studies

Faculty of Psychology

Educational and Scientific Center of Sociological Research of Precarpathian Region

Vasyl Stefanyk Precarpathian National University

57 Shevchenko Street

76018 Ivano-Frankivsk

Ukraine

e-mail: vnadurak@gmail.com

${ }^{1}$ Evans, Stanovich (2013): 223.
} 
dual-process theories. ${ }^{2}$ Although they are quite diverse, there are some typical characteristics that are mainly attributed to the respective kinds of information processing. The first (intuitive) is often described as fast, effortless (efficient), parallel, non-conscious, automatic, and associative. The second (deliberate) is described as slow, serial, conscious, controlled, rule-based, and effortful (inefficient). It should be emphasized that this set of characteristics is not canonical, but one that is typically encountered when it comes to these kinds of information processing.

The two kinds of thinking are also often referred to as System 1 and System 2. These terms were coined by Stanovich ${ }^{3}$ and have become extremely popular thanks to the works of Daniel Kahneman and his colleagues. ${ }^{4}$ Kahneman and Frederick described the interaction of these systems as follows: "System 1 quickly proposes intuitive answers to judgment problems as they arise, and System 2 monitors the quality of these proposals, which it may endorse, correct, or override. ${ }^{\prime 5}$ Although the terms System 1 and System 2 are very common, Evans and Stanovich argued against their use. They "prefer to avoid this terminology as it suggests (falsely) that the two types of processes are located in just two specific cognitive or neurological systems." ${ }^{1}$ Instead, they "reverted to the older terminology of Type 1 and 2 processing. These terms indicate qualitatively distinct forms of processing but allow that multiple cognitive or neural systems may underlie them." ${ }^{\prime 7}$

Despite their popularity, the dual-process theories have been also famously criticized.

For example, Gideon Keren wrote that "The main problem with all two-system theories, ... is that they are too broad, they fail to specify any clear constraints, and thus they are untestable." 8 Keren and Schul noted that "the different two-system theories lack conceptual clarity." 9

Melnikoff and Bargh suggested the so-called Good/Bad Fallacy inherent in dual-process theories which seem to claim that "Type 2 processing is good in that it generates rational judgments and decisions, whereas Type 1 processing is bad and error-prone." ${ }^{10}$ Kruglanski and Gigerenzer also charged the dual-process approach with the same fallacy while pointing out that, on the contrary, Type 1 processing can also give correct responses and Type 2 false ones. ${ }^{11}$ In reaction to this criticism, advocates of the dual-process approach agree that intuition "can automatically, quickly and effortlessly generate a skilled response to current challenges," thus denying that they interpret Type 1 processing as bad and error-prone. ${ }^{12}$

\footnotetext{
2 Evans, Stanovich (2013); Kahneman (2011); Kahneman, Frederick (2005); Sloman (2014).

${ }^{3}$ Stanovich (1999).

${ }^{4}$ Kahneman (2011); Kahneman, Frederick (2005).

${ }^{5}$ Kahneman, Frederick (2005): 267.

${ }^{6}$ Evans, Stanovich (2013): 225.

7 Ibidem: 226.

${ }^{8}$ Keren (2013): 260.

${ }^{9}$ Keren, Schul (2009): 535.

${ }_{10}$ Melnikoff, Bargh (2018): 282.

${ }^{11}$ Kruglanski, Gigerenzer (2011).

12 Pennycook, Neys, Evans et al. (2018): 667.
} 
Some researchers suggest that evidence can be taken to support quantitative differences rather than qualitatively distinct processes ${ }^{13}$ Accordingly, the differences between these processes can be described as a continuum. Yet, as Keren notes:

Both Newstead (2000) and Osman (2004) were correct when they asserted that demonstrations of processing continua undermine dual-process models - a point reiterated by Keren and Schul (2009). The logic is simple: If a particular dimension (e.g., automatic vs. controlled) is continuous, where is the cutting line that separates the two systems? This is a major drawback that makes the theory untestable. ${ }^{14}$

Claiming that there is no qualitative difference between the two types of processes, some researchers suggest replacing the dual-process (system) model with "single-system accounts," 15 or "a unified theoretical approach to both intuitive and deliberative judgments. Both are rule-based, and in fact, the very same rules can underlie both intuitive and deliberate judgments."16

One of the objects of criticism are the characteristics that are attributed to the two types of information processing. In particular, it is stated that "attribute clusters associated with dual systems do not consistently hold together." ${ }^{17}$ Melnikoff and Bargh called it "the Alignment Problem." They note:

For all we know, Type 1 features (e.g., unconsciousness) are no likelier to occur with other Type 1 features (e.g., unintentional) than they are to occur with Type 2 features (e.g., intentional). Likewise, it could be the case that Type 2 features (e.g., consciousness) are no likelier to occur with other Type 2 features (e.g., intentional) than they are to occur with Type 1 features (e.g., unintentional). The basic tenet of the Type $1 /$ Type 2 distinction - that the attributes within each category are aligned - simply has not been demonstrated. ... Yet, there are also many examples of where processing features do not align as the dual-process typology would predict. ${ }^{18}$

Similar criticism has also been expressed by Keren \& Schul ${ }^{19}$ and Kruglanski \& Gigerenzer. ${ }^{20}$

Responding to this criticism, a group of authors, including some of the most famous advocates of the dual-process model - Evans and Stanovich - noted that back in 2013, the latter "have explicitly argued against assuming an alignment of the numerous characteristics that have been assigned to so-called 'Type 1' and 'Type 2 '." ${ }^{21}$ Instead, they propose to distinguish between "defining features - those that are used to define

\footnotetext{
${ }^{13}$ Osman (2004); Kruglanski (2013).

${ }^{14}$ Keren (2013): 259.

${ }^{15}$ Osman (2004).

${ }^{16}$ Kruglanski, Gigerenzer (2011): 97.

${ }^{17}$ Evans, Stanovich (2013): 227.

${ }^{18}$ Melnikoff, Bargh (2018): 282.

${ }^{19}$ Keren, Schul (2009).

${ }^{20}$ Kruglanski, Gigerenzer (2011).

${ }^{21}$ Pennycook, Neys, Evans et al. (2018): 667.
} 
the two-types distinction - and typical correlates - those that various researchers have associated with the two-types distinction." 22

Despite these and other criticisms, the dual-process model has not lost its popularity and is widely used in the cognitive sciences. Its development continues, and its supporters seek to respond to critical remarks. In this article, I also want to provide such responses. My work is based on several assumptions.

First, it is worth noting that when Evans and Stanovich ${ }^{23}$ proposed replacing talk of two Systems with two Types of processes, they thus shifted the discussion towards the classification of different information processing processes.

Secondly, Evans and Stanovich's efforts to find definitive characteristics of these two types of processes can be interpreted as attempts to find criteria by which information processing could be classified so that the formed categories (types) were mutually exclusive and not overlapping. The latter is the basic condition for correct classification.

I wish to develop such an approach and therefore the purpose of this article is to propose a consistent classification of the two types of information processing, based on their definitive characteristics. It is hoped that a correctly performed classification will refute some of the criticisms against dual-process theories in particular, that it will prove qualitative rather than quantitative differences between the two types of processes, and that the formed types do not overlap.

\section{Information processing acts}

One of the objects of criticism of the dual-process theory is the fact that characteristics that are traditionally assigned to the different types, can be simultaneously inherent in one process. For example, Melnikoff and Bargh ${ }^{24}$ analyzing the well-known bat-and-ball problem note that the erroneous answer " 10 cents" is uncontrollable (this trait is attributed to processes of the first type) but the process by which we generate the uncontrollable error is intentional (a trait that is sometimes attributed to processes of the second type). ${ }^{25}$ They also point out that a process can have an unconscious cause and a conscious effect ${ }^{26}$ - two features that are attributed to the opposing types of processing.

Indeed, when characterizing certain processes, we often encounter cases when different parts of the same process have opposite characteristics. As an obvious example, consider the following inference:

Category $X$ drugs have many side effects.

Drug $Y$ belongs to category $X$.

Therefore, drug $Y$ has many side effects.

\footnotetext{
${ }^{22}$ Ibidem.

23 Evans, Stanovich (2013).

24 Melnikoff, Bargh (2018).

${ }^{25}$ Ibidem: 287.

26 Ibidem: 288.
} 
For instance, it is obvious that the first judgment may be intuitive for an experienced doctor and appear uncontrollable when this category of drugs is mentioned. At the same time, the second judgment and conclusion can be controlled when this doctor is faced with a new drug and finds out to which group it belongs. In this case, it is quite possible to agree with Thompson and Newman that every answer is produced by a combination of autonomous and controlled processes. ${ }^{27}$

However, each process is a series of actions. Hence, each process of information processing consists of a series of acts of information processing, that is, of combining two units of information. For example, the judgment "John is a good man" is the act of combining two units of information - "John" and the category of "good men."

Inference is a series of acts of information processing. For example:

All members of the ethnic group $X$ are thieves.

Person $Y$ is a member of the ethnic group $X$.

So, person $\mathrm{Y}$ is a thief.

Firstly, in this inference, each judgment is an act of combining units of information. In the first judgment, we combine the category "members of the ethnic group X"

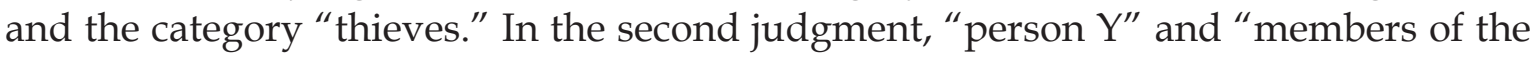
ethnic group $X$ " are combined. And in the third "person $Y^{\prime \prime}$ and "thieves."

Secondly, there is an indirect connection of units of information when by connecting the first unit (person $\mathrm{Y}$ ) with the second (members of the ethnic group X), we also indirectly connect it with the third unit (thieves).

Lastly, the acts that make up the inference may have opposite characteristics. For example, the first can be uncontrolled and fast, while the second and third are controlled and slower. Therefore, the process itself - inference - may consist of acts with opposite characteristics. Accordingly, it cannot be categorized unequivocally as controlled or uncontrolled, fast or slow.

However, it is quite obvious that a particular act of information processing at a particular moment in time cannot simultaneously have opposite characteristics (e.g., be fast and slow, given the same criterion of speed, or controlled and uncontrolled). That is why a particular act, not a process, of information processing should be classified. Otherwise, we will encounter cases where the same process has characteristics that are attributed to different types of information processing.

It should be emphasized that it is necessary to classify exactly how the units of information are combined, and not, for example, how this act was initiated. Since the act of initiating the combining of units of information and the actual act of combining are different acts; therefore, they may have opposite characteristics. For example, a stimulus may be unconscious and uncontrolled, while an act may be conscious and controlled. Therefore, this article will offer a classification of not just acts of information processing, but of how the combining of units of information occurs in them.

${ }^{27}$ Thompson, Newman (2018): 131. 


\section{Dichotomy}

One of the typical accusations against the dual-process approach is that the difference between the two types of information processing is quantitative rather than qualitative. For example, if we talk about the speed of information processing, it can vary from large (in the first type of processes) to small (in the second type). However, speed is inherent in both types, so it cannot be said that they are qualitatively different. The same goes for efficiency. If we take such features as a classification criterion, then we really do not find "the cutting line that separates the two systems," and this, as Gideon Keren says, "is a major drawback that makes the theory untestable." 28

To demonstrate a qualitative difference between the two types, it is necessary to show that one type has a certain quality, while the other does not - in other words, to make a dichotomous classification. For example, if we divide all acts of information processing into controlled and uncontrolled, then we get two categories, one of which includes acts with the trait "controllability", and the other those in which it is absent. Thus, we get two qualitatively different types and not just a continuum.

Of course, if we divide the acts of information processing, for example, into controlled and uncontrolled, then there will be a quantitative difference among the controlled acts - that is, there will be a continuum from weakly to strongly controlled acts. However, such a continuum will not create problems for a clear division into two types, because the qualitative difference between them will remain: acts of the first type will be uncontrolled, and the second controlled but to varying degrees.

To sum up, for the correct classification of information processing acts, it is necessary to make a dichotomous classification. It should be noted that this approach is often found in other sciences. For example, in biology, it is typical to divide organisms into two categories, one has certain feature and the other does not (e.g., vertebrates and invertebrates, prokaryote and eukaryote, etc.). I cannot agree with the authors who criticize the dual-process approach because it is supposedly caused by the human "penchant for binaries," 29 and even call it "The Two Types Bias," ${ }^{30}$ concluding: "The rise of the dual-process typology has been propelled not by scientific evidence but by the human tendency to be seduced by simplifying but baseless stereotypes." ${ }^{31}$ The dichotomy is a simple, logical, and common way of classification in science, not just a stereotype. It divides a set into elements that have or do not have a certain characteristic. If such a classification is accurate, then it should be accepted.

\section{Classification criterion}

In order to correctly classify the acts of information processing, the proposed categories must not overlap. The dual-process theory has been repeatedly accused of violating this rule: critics note that there are processes that have characteristics that are attributed

\footnotetext{
${ }^{28}$ Keren (2013): 259.

${ }^{29}$ Melnikoff, Bargh (2018).

30 Ibidem.

${ }^{31}$ Ibidem: 284.
} 
to both the first and second types. ${ }^{32}$ That is, the resulting categories are not mutually exclusive.

This problem occurs when a series of characteristics are ascribed to each process. At the same time, of course, one can agree with Evans and Stanovich, who, responding to such criticism, note that to distinguish two types, "the only thing needed is at least one dichotomous property that is necessary and sufficient." ${ }^{33}$ Therefore, they, following Stanovich and Toplak, ${ }^{34}$ distinguished defining features and incidental correlates of Type 1 and Type 2 processing. The affiliation of a process to the first or second type is determined by whether it has this defining feature. In their opinion, "the defining characteristic of Type 1 processes is their autonomy. They do not require 'controlled attention,' which is another way of saying that they make minimal demands on working memory resources." ${ }^{35}$ On the other hand, "a key defining feature of Type 2 processing the feature that makes humans unique - is cognitive decoupling: the ability to distinguish supposition from belief and to aid rational choices by running thought experiments." 36 Also, unlike Type 1 , Type 2 processing requires working memory. ${ }^{37}$

Although the arguments of Evans and Stanovich are quite convincing, their choice of definitive features seems problematic. In my opinion, such a choice violates the consistency principle - "whichever principle we choose, we should follow it consistently." 38 When classifying processes into autonomous ones and those characterized by cognitive decoupling, it is not clear which criterion is chosen for the classification; moreover, it may not be the only one. The point is that when we describe a process using the adjectives autonomous, controlled, fast, slow, effortful, effortless, etc., then we describe its qualities. "Cognitive decoupling", however, is not an adjective but a compound noun. It does not denote quality, but a certain mental mechanism that is involved in processes of the second type. Therefore, it is likely that when classifying processes into autonomous and those based on cognitive decoupling, not one but two classification criteria were used, and this violates the "consistent principle" rule.

This drawback is absent in the division of processes or acts of information processing into those that require working memory and do not require it. In this case, there is only one classification criterion - working memory. However, some researchers point out that working memory is used by processes of both types; that is, intuitive processes, to a small extent, also require it. For example, Keren writes that "it is agreed that reliance on WM is a matter of degree, as some processes use it more than others. This is incompatible with E\&S's suggestion to dichotomize the extent of WM reliance in order to distinguish between Type 1 and Type 2 processing." 39 Thompson and Newman also note that "all processes require some WM or attentional resources. In order to form the basis of a response, a Type 1 output requires at least a minimal endorsement from

\footnotetext{
32 Keren, Schul (2009); Kruglanski, Gigerenzer (2011); Osman (2004); Melnikoff, Bargh (2018).

33 Evans, Stanovich (2013): 228.

34 Stanovich, Toplak (2012).

35 Evans, Stanovich (2013): 236.

36 Ibidem: 236.

37 Ibidem: 225.

38 Kelley (2014): 14.

39 Keren (2013): 258.
} 
working memory (Kahneman, 2003)." 40 Finally, even Evans and Stanovich, describing the processes of the first type, noted that they "make minimal demands on working memory resources." 41 If we agree with this, then we should not take working memory as a classification criterion, because the difference between the two types will again be quantitative, not qualitative.

Supporters of the dual-process approach made other attempts to find a single defining characteristic of two types. For example, Thompson, appreciating the efforts of Evans and Stanovich to find a single defining characteristic for the two types of processes, wrote: "At least in terms of Type 1 processes, the move to a single characterizing feature provides valuable simplification and clarity. Autonomous processes are those whose execution is mandatory, given the presence of their triggering conditions. This is a clear cut and testable definition." ${ }^{42}$ In an effort to develop this approach, she notes: "If Type 1 processes are defined as autonomous, why not define Type 2 processes as their complement? That is, why not define Type 2 processes to be controlled processes that can, in principle, be initiated by the individual?" 43 However, after analyzing this possibility, she finally concludes that the fact that Type 2 processes "may be initiated automatically suggests that control, though a strong coexisting feature of Type 2 processes, should not be considered a defining feature." ${ }^{44}$

Pennycook agrees with Thompson that autonomy is the only feature needed to distinguish the two types of processes. ${ }^{45}$ But, in his opinion, "the concept of autonomy naturally leads to questions about the potential source of the cognitive output. For an autonomous intuitive response, the answer to the question is straightforward: the (proximal) source of the response is the stimulus-response pairing(s)." 46

In his recent works, Evans ${ }^{47}$ decided to supplement his and Stanovich's conclusions about the definitive features of the two types of processes. He noted: "If I am relatively sanguine about the definition of Type 2 processing offered by Evans and Stanovich (2013), I cannot really say the same about Type 1 processes. These are defined essentially by exclusion. Type 1 processes are autonomous, meaning that they are not consciously controlled or engaging working memory." 48 The problem here is that this definition is too broad since there are numerous kinds of autonomous processes in the brain, including those that are not related to cognition (e.g., visual perception). Thus, he decides "to add something else to the definition of Type 1 processing as applied in dual process accounts of reasoning and decision making" - "feeling of knowing or correctness" 49 or "feeling of confidence or rightness" 50 associated with intuitive judgments. The presence of such feeling in his opinion is also a defining feature of intuitive processing. ${ }^{51}$

\footnotetext{
40 Thompson, Newman (2018): 132.

${ }^{41}$ Evans, Stanovich (2013): 236.

42 Thompson (2013): 253.

${ }^{43}$ Ibidem.

${ }^{44}$ Ibidem: 256.

45 Pennycook (2018): 7.

${ }^{46}$ Ibidem: 9.

47 Evans (2018); Evans (2019).

${ }^{48}$ Evans (2018): 142.

49 Ibidem.

${ }^{50}$ Evans (2019).

${ }^{51}$ Evans (2018): 142.
} 
In the next section, I will offer my thoughts on the definitive feature that would allow us to classify acts of information processing into two types. Evans wrote that "Type 1 processes are autonomous, meaning that they are not consciously controlled or engaging working memory." 52 I find this characteristic very precise, so I will use it as a basis for my approach. However, given the above remarks about working memory, it will not be used in my definition. Accordingly, the acts of information processing of the first type will be defined as autonomous, and of the second type - as consciously controlled. In this case, conscious control will be accepted as a criterion for classifying information processing acts into two types.

It should be noted that the distinction between controlled and automatic processing has had a long history in the literature on attention, ${ }^{53}$ which had a big impact on the dual process theory. Therefore, the proposed classification is not new, but the arguments in its favor can be considered new.

\section{Conscious control}

Overall, I agree with Evans and Stanovich, ${ }^{54}$ Thompson, ${ }^{55}$ Pennycook, ${ }^{56}$ and others, who argue that autonomy is a good candidate for the defining feature for Type 1 processing. I also agree with Thompson ${ }^{57}$ that if Type 1 processes are defined as autonomous, then it is worth considering the possibility to define Type 2 processes as their complement, that is, defining Type 2 processes to be controlled processes. Yet, I cannot agree with her final conclusion, that the fact that Type 2 processing "may be initiated automatically suggests that control, though a strong coexisting feature of Type 2 processes, should not be considered a defining feature." ${ }^{58}$ However, before finally defining the acts of information processing of the first type as autonomous, and the second as controlled, it is necessary to make some clarifications in order to fulfill the requirements for the correct classification.

First, it should be clearly stated that it is conscious control that is the criterion for dividing the set of acts of information processing into two types. Accordingly, in an effort to carry out a dichotomous classification, when we refer to Type 2 acts of information processing, we mean those that are consciously controlled. It should be noted that it is necessary to talk specifically about consciously controlled acts, since other acts of information processing, which are often viewed as uncontrolled, can actually be interpreted as controlled, not by consciousness, but by the parts of our mind that work unconsciously. An example of such an act could be an instant decision to take a hand away from a hot object. It is obvious that it is not consciously controlled, but it is also obvious that it is controlled outside of our consciousness, by other parts of our brain.

\footnotetext{
52 Evans (2018): 142.

53 Schneider, Shiffrin (1977); Shiffrin, Schneider (1977).

54 Evans, Stanovich (2013).

55 Thompson (2013).

56 Pennycook (2017).

57 Thompson (2013).

58 Ibidem: 256.
} 
Second, acts of information processing of the first type will be defined "essentially by exclusion." 59 That is, if acts of the second type are consciously controlled, then the first type includes those that are not consciously controlled. In other words, they can be called autonomous, which corresponds to the literal meaning of this word: "The word autonomous (deriving from the Greek words auto [self] and nomos [law]) literally means self-governed or not controlled by outside forces." 60

Performing such a classification, we divide the entire set of acts of information processing into two categories: one includes acts that have a certain characteristic (conscious control), and the other in which the characteristic is absent. The resulting categories do not overlap and between them there is a clear "cutting line." 61 Thus, we show that there is not just a quantitative difference between the two types of acts but also a qualitative one, refuting the accusations of those who claim the opposite and defend the idea of the continuum.

At this point, it should be recalled that the object of classification in this article is how the combining of units of information occurs and not how this act was initiated. That is why I do not agree with Thompson that the fact that Type 2 processing "may be initiated automatically suggests that control, though a strong coexisting feature of Type 2 processes, should not be considered a defining feature." ${ }^{62}$ As an example, she cites unusual situations that can be "an autonomous trigger to Type 2 thinking, such as when one confronts a bizarrely dressed person in the mail room..." ${ }^{33}$ However, let's imagine that we found ourselves in such a situation and made a controlled conclusion - "this person is a comedian." If in this case we classify not how this judgment was initiated, but how the combining of the two units of information ("this person" and "comedian") occurred, then the problem disappears and we can unequivocally assert that this act belongs to the second type of information processing.

Of course, the two categories of information processing acts that we have identified can be characterized in more detail by attributing other characteristics to them. This, in turn, may become the basis for identifying their subcategories. For example, autonomous acts can be divided into those that are accompanied and not accompanied by "feeling of confidence or rightness." ${ }^{64}$ However, I am not going to develop this issue here, fully agreeing with Evans and Stanovich, ${ }^{65}$ that even one definitive characteristic is enough to show the existence of two qualitatively different types of information processing.

Although these clarifications make it possible to make a logically correct classification, the question still remains, why it is conscious control that was chosen as a classification criterion. To explain this, it is necessary to consider the concepts of connected and unconnected units of information.

\footnotetext{
${ }^{59}$ Evans (2018).

${ }^{60}$ Moors, De Houwer (2006): 307.

${ }^{61}$ Keren (2013).

${ }^{62}$ Thompson (2013): 256.

${ }^{63}$ Ibidem: 255.

${ }^{64}$ Evans (2019).

${ }^{65}$ Evans, Stanovich (2013).
} 
Let me remind the reader that each act of information processing is an act of combining certain units of information. For example, the judgment "person X is a liar" is a combination of two units of information - "person X" and the predicate "liar."

Some units of information do not need to be combined, because they are already connected in our mind and the appearance of one automatically invokes the other; that is, there is an associative connection between them (it should be noted that Morewedge and Kahneman identify processes of the first type "with the automatic operations of associative memory" 66 and I agree with them). An example would be the question "How much is twice two" and the answer "Four." When these units are connected in our memory, the appearance of one automatically causes the appearance of another. Accordingly, we do not need to consciously and in a controlled manner combine them.

However, if we take the question "How much is $2 \times 22341$ " and the answer "44682" as an example, then it should be noted that in the minds of most of us these two units of information are not joined into a ready-made combination that is stored in long-term memory. To connect them, you need to perform certain operations in working memory. This action is impossible without conscious control. That is why this way of combining units of information is a defining characteristic that distinguishes two types of information processing acts.

It should be noted that unconnected units of information could sometimes be combined without conscious control. This is possible due to automatic attribute substitution - "the mechanism that generates heuristic judgments, in which the answer to a simpler (and more accessible) question is substituted for a difficult one." ${ }^{67}$ For example, "the instruction to evaluate whether a person is generous will automatically evoke judgment of that person on other favorable dimensions (e.g., whether she is warm, friendly, or virtuous)." ${ }^{68}$ If in our memory this person is associated with the characteristic friendly, then it is quite possible that it will automatically connect with the characteristic generous. Thus, we will give an affirmative answer to the above question, although we do not really know whether this person is generous; this "substitution typically occurs without any awareness." ${ }^{69}$

A possible explanation for this substitution is that in our minds many positive characteristics are associated with each other. That is, for example, the characteristics warm, friendly, virtuous, and generous are connected units of information. Accordingly, if information about person $\mathrm{X}$ is connected with the characteristic friendly, and this characteristic, in turn, is connected with some other characteristics, including generous, then the attribute substitution occurs as a combining of information units "person $X$ " and "generous" through the mediation of the characteristic friendly. In this case, we will have an example of an autonomous act of information processing.

To summarize, we can say that in the second type of information processing acts, units of information are combined in a consciously controlled way, and in the first type they either already are combined, or combine autonomously due to the existence of indirect associative connections.

\footnotetext{
${ }^{66}$ Morewedge, Kahneman (2010): 435.

${ }^{67}$ Ibidem: 437.

${ }^{68}$ Ibidem.

${ }^{69}$ Ibidem.
} 


\section{Final remarks}

It is worth mentioning a few more theses that will supplement what has been written and responses to possible criticism.

First, if we want to create a unified theory that would claim to be universally accepted, then as a classification criterion it is worth taking a certain fundamental feature that would allow not only to divide the set into subsets, but also to explain the phenomena belonging to it (in particular, explain other features attributed to them). I think that such a characteristic as conscious control performs this role well. For example, combining units of information in a consciously controlled way usually requires more time and effort than when these units are combined autonomously or are already combined. This type of information processing acts is sequential since only one act can be consciously controlled at a time. So, at least some of the features that are mainly attributed to acts of the second type can be explained based on conscious control. This gives grounds to consider this feature as fundamental for the information processing acts of the second type.

Secondly, it is necessary to respond to the claim of critics of the dual-process approach that

The distinction between automatic and controlled behavior, in this framework, is unequivocally on a continuum. To illustrate, consider driving behavior that can be described initially as pure controlled behavior, which, with practice (over months and years), gradually requires fewer and fewer resources and thus turns into an automatic activity. ${ }^{70}$

It should be noted here that on a continuum is a distinction not between automatic and controlled behavior, but between different types of controlled behavior. Namely, there is a quantitative difference among controlled acts, a continuum from weakly controlled to strongly controlled acts. However, autonomous acts occur without conscious control. Therefore, they are not part of this continuum, since "a continuum is a set of things on a scale, which have a particular characteristic to different degrees." ${ }^{71}$ But autonomous acts do not possess this characteristic - conscious control - to any degree. Thus, the above critical remark cannot be considered relevant when it comes to the difference between autonomous and consciously controlled acts of information processing.

Lastly, it is necessary to consider the statement of Keren and Schul, who, criticizing the dual-system approach, noted that "dichotomizing implies oversimplifying."72 Of course, we can agree that the dichotomizing offered by the dual-type approach simplifies the extremely complex picture of cognitive processes. But is this oversimplification? Is it an oversimplification to divide the extremely rich animal world into vertebrates and invertebrates, prokaryotes and eukaryotes? Perhaps so. However, science needs such simplifications. Thanks to them, we organize and partially explain a set of complex

\footnotetext{
70 Keren, Schul (2009): 538.

${ }^{71}$ Collins Online Dictionary (2020).

${ }^{72}$ Keren, Schul (2009): 546.
} 
phenomena, which is a necessary step at a certain stage of development in a particular field of knowledge.

Does such simplification come at a price? Yes. For example, such a dichotomy may lead us to exaggerate the difference between acts belonging to different types. On the other hand, the proposed dichotomy does not show the difference that exists between acts belonging to the same type. For instance, when we talk about the category of consciously controlled information processing acts, we must remember that they sometimes differ significantly from each other both in the amount of conscious control and in other correlative features such as speed, efficiency, and the like. Similarly, there is a difference between autonomous acts regarding their origin (i.e., innate versus acquired), or how rational they are. Therefore, the dichotomy between the two types of acts highlighted in this article is only one of the steps that are designed to prove the qualitative difference between them. The description of different variations of these types is another essential part of a viable theory of the two types of information processing acts.

Consequently, it would not be superfluous to emphasize once again that to prove the existence of two qualitatively different types of information processing acts, one characteristic is sufficient. However, in order to describe these types, other characteristics are needed.

In conclusion, it should be noted that the ease with which we can logically separate the two types of information processing acts should not lead us to think that it will be possible to achieve such ease with real phenomena. It is sometimes difficult to determine whether a particular act is consciously controlled or not. The point is that this very characteristic - conscious control - is gradual. A feature is gradual when it can be present to some degree. ${ }^{73}$ Accordingly, in some information processing acts, the degree of conscious control may be so insignificant that its detection will be an almost impossible task. However, this is a problem with the methods we use, and it cannot disprove the conclusion that there are two types of information processing acts, one of which is characterized by conscious control, and the other is not.

\section{Conclusion}

This article proposes a classification of different types of information processing, which would be resistant to the main criticisms presented to the dual-process theory.

In order to complete the task, it was necessary to determine the object of classification. This article proposes to classify not the process of information processing, but acts of combining two units of information. This is due to the fact that a single process can sometimes have characteristics which are attributed to the opposite types of information processing (critics of the dual-process approach have repeatedly drawn attention to this problem). Instead, a particular act of information processing at a particular moment in time cannot simultaneously have opposite characteristics. Accordingly, by classifying acts, the above criticism can be avoided.

${ }^{73}$ Moors, De Houwer (2006): 321. 
One of the typical accusations against the dual-process approach is that the difference between the two types of information processing is quantitative rather than qualitative; that is, there is a continuum between them. Therefore, in order to show the qualitative difference between the two types, it is necessary to classify not by a feature present to varying degrees in the two types, but by a feature that is present in one type and absent in another. It is suggested to take conscious control as such a feature. Accordingly, the whole set of information processing acts can be divided into two categories: one of them (Type 2) includes acts that have this feature (conscious control), and the other (Type 1) those in which it is absent (they can be called autonomous). Thus, we show that there is not just a quantitative difference between the two types of acts but also a qualitative one, refuting the accusations of those who claim the opposite and defend the idea of the continuum.

The choice of conscious control as a criterion for classification is due not only to formal reasons - to make a logically correct classification. This choice is also based on the vision of cognition as a combining of units of information. Some units are already connected in our mind and the appearance of one automatically invokes the other. But other units of information need to be connected. This can be done by combining them in working memory in a consciously controlled way. That is why this way of combining is a defining characteristic that distinguishes two types of information processing acts.

It should be noted that, although the proposed classification well fulfills the purpose of the article - to prove the existence of two qualitatively different types of information processing acts - it should not be considered exhaustive. Further work should be aimed at describing the varieties of these two types. Finally, other attempts to classify acts of information processing based on other criteria can be made. Therefore, new research in this field has every chance to be quite productive.

Acknowledgments. I would like to thank the anonymous reviewer and Jonathan Evans for their valuable comments. Also, my sincere thanks to Stephanie Young for her support and proofreading of this article.

\section{Bibliography}

Collins Online Dictionary (2020), “Continuum,” URL = https:/ /www.collinsdictionary.com/ dictionary/english/continuum [Accessed 01.02.2021].

Evans J.S.B.T. (2019), “Reflections on Reflection: The Nature and Function of Type 2 Processes in Dual-Process Theories of Reasoning," Thinking \& Reasoning 25 (4): 383-415.

Evans J.S.B.T. (2018), “Dual Process Theory: Perspectives and Problems,” [in:] Current Issues in Thinking and Reasoning. Dual Process Theory 2.0, W. De Neys (ed.), Routledge/Taylor \& Francis Group, London, New York: 137-155.

Evans J.S.B.T., Stanovich K.E. (2013), “Dual-Process Theories of Higher Cognition: Advancing the Debate," Perspectives on Psychological Science 8 (3): 223-241.

Kahneman D. (2003), "A Perspective on Judgment and Choice: Mapping Bounded Rationality," American Psychologist 58 (9): 697-720.

Kahneman D. (2011), Thinking Fast and Slow, Farrar, Straus \& Giroux, New York. 
Kahneman D., Frederick S. (2005), "A Model of Heuristic Judgment," [in:] The Cambridge Handbook of Thinking and Reasoning, K.J. Holyoak, R.G. Morrison (eds.), Cambridge University Press, Cambridge (UK): 267-293.

Kelley D. (2014), The Art of Reasoning: An Introduction to Logic and Critical Thinking (Fourth Edition), W.W. Norton \& Company, New York, London.

Keren G. (2013), "A Tale of Two Systems: A Scientific Advance or a Theoretical Stone Soup? Commentary on Evans \& Stanovich," Perspectives on Psychological Science 8 (3): 257262.

Keren G., Schul Y. (2009), “Two is Not Always Better Than One: A Critical Evaluation of Two-System Theories," Perspectives on Psychological Science 4 (6): 533-550.

Kruglanski A.W., Gigerenzer G. (2011), “Intuitive and Deliberate Judgments Are Based on Common Principles," Psychological Review 118 (1): 97-109.

Kruglanski A.W. (2013), “Only One? The Default Interventionist Perspective as a Unimodel-Commentary on Evans \& Stanovich," Perspectives on Psychological Science 8 (3): 242-247.

Moors A., De Houwer J. (2006), "Automaticity: A Theoretical and Conceptual Analysis," Psychological Bulletin 132 (2): 297-326.

Morewedge C.K., Kahneman D. (2010), “Associative Processes in Intuitive Judgment," Trends in Cognitive Sciences 14 (10): 435-440.

Newstead S.E. (2000), "Are There Two Different Types of Thinking?," Behavioral and Brain Sciences 23 (5): 690-691.

Osman M. (2004), "An Evaluation of Dual-Process Theories of Reasoning," Psychonomic Bulletin \& Review 11 (6): 988-1010.

Osman M. (2013), "A Case Study: Dual-Process Theories of Higher Cognition-Commentary on Evans \& Stanovich," Perspectives on Psychological Science 8 (3): 248-252.

Pennycook G. (2018), "A Perspective on the Theoretical Foundation of Dual Process Models," [in:] Current Issues in Thinking and Reasoning. Dual Process Theory 2.0, W. De Neys (ed.), Routledge/Taylor \& Francis Group, London, New York: 5-27.

Pennycook G., Neys W., Evans J. et al. (2018), “The Mythical Dual-Process Typology,” Trends in Cognitive Sciences 22 (8): 667-668.

Schneider W., Shiffrin R.M. (1977), "Controlled and Automatic Human Information Processing: I. Detection, Search, And Attention," Psychological Review 84 (1): 1-66.

Shiffrin R.M., Schneider W. (1977), "Controlled and Automatic Human Information Processing: II. Perceptual Learning, Automatic Attending and a General Theory," Psychological Review 84 (2): 127-190.

Sloman S. (2014), "Two Systems of Reasoning: An Update," [in:] Dual-Process Theories of the Social Mind, J.W. Sherman, B. Gawronski, Y. Trope (eds.), The Guilford Press, New York: 69-79.

Sloman S.A. (1996), “The Empirical Case for Two Systems of Reasoning," Psychological Bulletin 119 (1): 3-22.

Stanovich K.E. (1999), Who is Rational?: Studies of Individual Differences in Reasoning, Lawrence Erlbaum Associates Publishers, Mahwah (New Jersey).

Stanovich K.E., Toplak M.E. (2012), “Defining Features Versus Incidental Correlates of Type 1 and Type 2 Processing," Mind \& Society 11 (1): 3-13.

Stanovich K.E., West R.F. (2002), “Individual Differences in Reasoning: Implications for the Rationality Debate?," [in:] Heuristics and Biases: The Psychology of Intuitive Judgment, T. Gilovich, D. Griffin, D. Kahneman (eds.), Cambridge University Press, Cambridge (UK), New York: 421-440. 
Thompson V.A. (2013), “Why it Matters: The Implications of Autonomous Processes for Dual Process Theories - Commentary on Evans \& Stanovich," Perspectives on Psychological Science 8 (3): 253-256.

Thompson V.A., Newman I.R. (2018), “Logical Intuitions and Other Conundra for Dual Process Theories," [in:] Current Issues in Thinking and Reasoning. Dual Process Theory 2.0, W. De Neys (ed.), Routledge/Taylor \& Francis Group, London, New York: 121-136. 